\title{
Exposição humana ao metilmercúrio em comunidades ribeirinhas da Região do Tapajós, Pará, Brasil
}

\author{
Methylmercury human exposure in riverine villages of \\ Tapajos basin, Pará State, Brazil
}

\author{
Maria da Conceição Nascimento Pinheiro', Junko Nakanishi², Teichii Oikawa', Geraldo \\ Guimarães 1 , Manoel Quaresma1, Bernardo Cardoso', Walter W. Amoras', Masazume Harada ${ }^{3}$, \\ Carlos Magno ${ }^{4}$, José Luis F. Vieira ${ }^{1}$, Marilia Brasil Xavier ${ }^{1}$ e Denise R. Bacelar ${ }^{1}$
}

\begin{abstract}
Resumo Avaliou-se a exposição humana ao metilmercúrio e ao mercúrio total em comunidades ribeirinhas do rio Tapajós e da região metropolitana de Belém, no Estado do Pará, Brasil, através da determinação de mercúrio total e metilmercúrio em amostras de cabelo nos anos de 1994 e 1995. Observou-se que as concentrações médias de mercúrio total variaram de $2 \pm 1 \mu \mathrm{g} / \mathrm{g}^{-1}$ a $20,5 \pm 12,1 \mu \mathrm{g} / \mathrm{g}^{-1}$, enquanto que as concentrações médias de metilmercúrio variaram de $1,4 \pm 0,7 \mu \mathrm{g} / \mathrm{g}^{-1}$ a 18,5 $\pm 11 \mu \mathrm{g} / \mathrm{g}^{-1}$. Estes resultados confirmam a contaminação mercurial na região do rio Tapajós, admitem a possibilidade do aparecimento de sinais e sintomas de intoxicação mercurial e recomendam a manutenção da monitorização do mercúrio total e do metilmercúrio nas amostras de cabelo, bem como a necessidade de estudos clínico-epidemiológicos para implantação de medidas de prevenção e controle da intoxicação mercurial.
\end{abstract}

Palavras-chaves: Metilmercúrio. Contaminação mercurial. Poluição ambiental. Rio Tapajós.

Abstract Evaluation of total human exposure to mercury and methylmercury was effected in riverine villages along the Tapajos river and in the metropolitan area of Belem city, state of Para, Brazil, by using total mercury and methylmercury concentrations in hair samples in 1994 and 1995. It was observed that average concentrations of total mercury are in a range from $2 \pm 1 \mu \mathrm{g} / \mathrm{g}^{-1}$ to $20.5 \pm 12.1 \mu \mathrm{g} / \mathrm{g}^{-1}$. While methylmercury average concentration varies from $1.4 \pm 0.7 \mu \mathrm{g} / \mathrm{g}^{-1}$ to $18.5 \pm 11 \mu \mathrm{g} / \mathrm{g}^{-1}$. These results confirm mercury contamination in the Tapajos river and possible appearance of mercury intoxication symptoms, and recommends the monitoring of compounds in hair samples as well as the need for epidemiological and clinical studies for human health prevention and control of mercury intoxication.

Key-words: Methylmercury. Mercury contamination. Environmental pollution. Tapajos river.

A exposição humana ao metilmercúrio através da ingestão de alimentos contaminados tem sido associada a tragédias ambientais, em diferentes partes do mundo, como conseqüência principalmente das atividades industriais ${ }^{1211617}$.
Hoje são conhecidos importantes acidentes ambientais decorrentes da descarga de resíduos industriais contendo mercúrio, em baías, rios e lagos, contaminando animais de vida marinha e secundariamente as populações humanas ${ }^{61315}$.

\footnotetext{
1. Núcleo de Medicina Tropical da Universidade Federal do Pará, Belém, PA, Brasil. 2. Institute of Environmental Science and Technology Yokohama National University. 3. Institute of Molecular Embryology and Genetics Scholl of Medicine of Kumamoto University. 4. Departamento Nacional de Produção Mineral.

Endereço para correspondência: Dra. Maria da Conceição Nascimento Pinheiro. Núcleo de Medicina Tropical/UFPA. Av. Generalíssimo Deodoro 92, Umarizal, 66055-240 Belém, PA, Brasil.

Tel: 5591 241-9864/0032.

Recebido para publicação em 17/11/98.
} 
A contaminação mercurial dos rios e lagos decorrentes das atividades garimpeiras de ouro, com conseqüente contaminação dos peixes e humanos, tem sido caracterizada na região Amazônica brasileira127911 .

Na Região do Rio Tapajós, a contaminação pelo mercúrio, vem sendo estudada através da análise do metal nos peixes e nas amostras de cabelo das populações de diferentes comunidades ribeirinhas ${ }^{2}$.

Sabe-se que, no processo da garimpagem do ouro nos rios da Amazônia, grande quantidade de mercúrio é utilizada na captação de finas partículas do metal nobre, formando um amálgama ouromercúrio que posteriormente é submetido a queima para separação dos metais. $O$ excesso do mercúrio líquido e o vapor resultante da queima do amálgama, depositam-se nos rios e lagos da região, onde provavelmente sofrem processos de metilação, com conseqüente acúmulo na cadeia alimentar aquática ${ }^{4} 91117$.

Estudos demonstraram que determinadas espécies de peixes da região, consideradas de hábito carnívoro, como Hoplias malabarilus (traíra), Cichla ocellaris (tucunaré), Plagioscion surinamensis (pescada), Hydrolycus scomberoides (peixe cachorro), dentre outras, freqüentemente consumidos pela população local, apresentaram níveis de mercúrio total acima do limite recomendável para consumo humano pela Organização Mundial da Saúde, isto é, superiores a $0,5 \mu \mathrm{g} / \mathrm{g}^{-112}$.

A exposição humana ao metilmercúrio tem sido avaliada pela determinação das suas concentrações em amostras de cabelo, que constitui, até o momento, um bom indicador biológico para avaliação desta exposição ${ }^{15} 17$

Visando contribuir para o controle da contaminação ambiental e como conseqüência prevenir a intoxicação pelo metilmercúrio, os autores avaliaram os níveis da contaminação mercurial humana em comunidades situadas às margens do rio Tapajós no Estado do Pará, no período de 1994 a 1995, através da determinação de mercúrio total e metilmercúrio em amostras de cabelo da população ribeirinha e compararam os resultados, com aqueles oriundos de população residente na região metropolitana de Belém, PA.

\section{MATERIAL E MÉTODOS}

Área estudada e características das comunidades. As áreas de estudo foram duas comunidades de pescadores (Rainha e Barreiras) localizadas às margens do Rio Tapajós, próximas à cidade de Itaituba no Estado do Pará, situada a $600 \mathrm{~km}$ da cidade de Belém, na qual, foram coletadas as amostras da população urbana.

As comunidades de pescadores são áreas de elevada carência sócio-econômica, cuja alimentação diária é constituída basicamente de peixes oriundos dos rios e igarapés que os cercam. Os participantes deste estudo são pescadores, lavradores e familiares que não apresentam relato de participação em atividade garimpeira.

As amostras coletadas na cidade de Belém foram oriundas de voluntários dos sexos masculino e feminino de poder sócio-econômico médio, que não desenvolviam nenhuma atividade relacionada ao uso do mercúrio e seus compostos e com ingestão média de uma refeição semanal a base de peixes.

Amostras. Foram obtidas aproximadamente $5 \mathrm{~g}$ de cabelo de cada voluntário participante do estudo, retirados cerca de $1 \mathrm{~cm}$ de distância de sua inserção, de três locais diferentes do couro cabeludo. A seguir, as amostras coletadas foram acondicionadas em envelope de papel, devidamente identificado.

No ano de 1994, foram coletadas 16 amostras de cabelo na comunidade de Rainha, 26 amostras em Barreiras e 13 amostras na região metropolitana de Belém.

No ano de 1995, foram coletadas 13 amostras em Rainha e 52 amostra em Barreiras.

Procedimento analítico. A determinação de mercúrio total em amostras de cabelo foi realizada por espectrofotometria de absorção atômica, com amalgamação em lâmina de ouro, empregandose monitor de mercúrio da Nippon Instrument Cooperation, modelo SP-3D.

A determinação de metilmercúrio nas amostras de cabelo foi realizada por cromatografia gasosa, empregando-se detetor de captura de elétrons em cromatógrafo Shimadzu.

Avaliação estatística. Na comparação dos resultados obtidos da determinação de mercúrio total e metilmercúrio nas amostras de cabelo das populações estudadas, empregou-se o teste $t$ de Student e a análise de variância (ANOVA) considerando-se $p<0,05$, com significância estatística. 
Parecer de ética. O presente estudo obteve aprovação do Comitê de Ética do Hospital João de Barros Barreto em 1994.

Ressalte-se que para obtenção das amostras de cabelo, o voluntário era informado e esclarecido sobre o objetivo da coleta. Após conceder autorização (em caso de crianças a autorização era fornecida pelos pais ou responsável), procedia-se a coleta das amostras.

\section{RESULTADOS}

As concentrações médias de mercúrio total e de metilmercúrio nas amostras de cabelo coletadas em comunidades ribeirinhas, localizadas às margens do rio Tapajós, nos anos de 1994 e 1995, juntamente com os valores obtidos na região metropolitana de Belém, no ano de 1995, encontramse descritos na Tabela 1.

Na comunidade de Rainha, observou-se que as concentrações médias de mercúrio total nas amostras de cabelo foram 19,3 $\pm 9,7 \mu \mathrm{g} / \mathrm{g}^{-1}$ em 1994 e $15,9 \pm 6,2 \mu \mathrm{g} / \mathrm{g}^{-1}$ em 1995. Enquanto as concentrações médias de metilmercúrio foram $18,3 \pm 8,5 \mu \mathrm{g} / \mathrm{g}^{-1}$ e $14,3 \pm 6,1 \mu \mathrm{g} / \mathrm{g}^{-1}$, nos anos de 1994 e1995, respectivamente.

Comparando-se os resultados da determinação de mercúrio total e metilmercúrio nas amostras de cabelo da comunidade de Rainha, obtidos no ano de 1994 e de 1995, não se observou diferença significativa no período de estudo $(T=0,9097$ $\mathrm{p}<0,05)$.
$\mathrm{Na}$ comunidade de Barreiras, as concentrações médias de mercúrio total nas amostras de cabelo analisadas no ano de 1994 foram 20,5 $\pm 12,1 \mu \mathrm{g} / \mathrm{g}^{-1}$, e no ano de 1995 foram $17,7 \pm 12 \mu \mathrm{g} / \mathrm{g}^{-1}$. Enquanto que as concentrações médias de metilmercúrio no ano de 1994 foram $18,5 \pm 11 \mu \mathrm{g} / \mathrm{g}^{-1}$ e em 1995 foram $15,8 \pm 1,6 \mu \mathrm{g} / \mathrm{g}^{-1}$.

Comparando-se os resultados da determinação de mercúrio total e metilmercúrio nas amostras de cabelo da comunidade de Barreiras, obtidos no ano de 1994 e de 1995 não se observou diferença significativa no período de estudo $(T=0,8208$ $\mathrm{p}<0,05)$.

$\mathrm{Na}$ região metropolitana de Belém as concentrações médias de mercúrio total e metilmercúrio nas amostras de cabelo coletadas em 1994, foram $2 \pm 1 \mu \mathrm{g} / \mathrm{g}^{-1}$ e $1,4 \pm 0,7 \mu \mathrm{g} / \mathrm{g}^{-1}$, respectivamente.

Tabela 1 - Resultados da determinação de mercúrio total e metilmercúrio em amostras de cabelo oriundas de comunidades ribeirinhas e de Belém.

\begin{tabular}{lcccccc}
\hline Localidade & №* & Ano & $\begin{array}{c}\text { Idade média } \\
\text { (anos) }\end{array}$ & $\begin{array}{c}\text { Concentração média } \\
\text { de mercúrio total } \\
\left(\mu \mathrm{g} / \mathrm{g}^{-1}\right) \text { x+d.p. }{ }^{* *}\end{array}$ & $\begin{array}{c}\text { Concentração média } \\
\text { de metilmercúrio } \\
\left(\mu \mathrm{g} / \mathrm{g}^{-1}\right) \text { x+d.p. }{ }^{* *}\end{array}$ & $\begin{array}{c}\text { Relação entre as concentrações } \\
\text { médias de mercúrio total } \\
\text { e metilmercúrio }(\%)\end{array}$ \\
\hline Rainha & 16 & 1994 & 26 & $19,3 \pm 9,7$ & $18,3 \pm 8,5$ & $96,0 \pm 4,6$ \\
Rainha & 13 & 1995 & 31 & $15,9 \pm 6,2$ & $14,3 \pm 6,1$ & $89,1 \pm 3,8$ \\
Barreiras & 26 & 1994 & 37 & $20,5 \pm 12,1$ & $18,5 \pm 11,0$ & $90,2 \pm 5,3$ \\
Barreiras & 52 & 1995 & 28 & $17,7 \pm 120$ & $15,8 \pm 1,6$ & $87,9 \pm 6,6$ \\
Belém & 13 & 1994 & 28 & $2,0 \pm 1.0$ & $1,4 \pm 0,7$ & $78,4 \pm 29,3$ \\
\hline
\end{tabular}

* número de determinações realizadas

** média e desvio padrão

\section{DISCUSSÃO}

A exposição humana ao metilmercúrio em comunidades de pescadores e em não ribeirinhos do rio Tapajós, situadas às proximidades de garimpos de ouro, vem sendo avaliada, através da determinação das concentrações do composto em peixes consumidos na região e nas amostras de cabelo da população ${ }^{125}$.
Embora o processo de metilação do mercúrio metálico no rio Tapajós não esteja totalmente esclarecido, sabe-se que o mercúrio inorgânico pode sofrer biometilação no meio aquático contaminando a cadeia alimentar, desde o fitoplâncton até o topo da cadeia representado pelos peixes carnívoros, onde estão as maiores concentrações do composto ${ }^{149}$. 
Nas comunidades ribeirinhas estudadas, não foram observadas diferenças significativas na contaminação mercurial humana no período de estudo, pois, ao se comparar as concentrações médias de mercúrio total nas comunidades de Barreiras e Rainha, no ano de 1994, não se observou diferença estatística significativa $(T=0,0790)$, a mesma observação foi detectada no ano de 1995 $(T=0,4567)$. Ressalte-se que as concentrações de mercúrio total nas amostras de cabelo destas populações encontraram-se acima de $6 \mu \mathrm{g} / \mathrm{g}^{-1}$, que é considerado o limite máximo de segurança ${ }^{16}{ }^{17}$.

As concentrações médias de mercúrio total e metilmercúrio nas comunidades de Rainha e Barreiras foram superiores aquelas determinadas na região metropolitana de Belém, sugerindo maior exposição das populações ribeirinhas ao metal e seu composto.

Percebeu-se que a porcentagem de metilmercúrio em relação ao mercúrio total nas amostras analisadas foi maior nas comunidades ribeirinhas quando comparadas àquelas coletadas em Belém.

As concentrações médias de mercúrio total nas amostras de cabelo das comunidades deste estudo são comparáveis àquelas observadas em outras comunidades de pescadores do rio Tapajós ${ }^{128}$.

A intoxicação por metilmercúrio ocorrida em Minamata, no Japão, só foi definitivamente confirmada vários anos após a exposição, quando muitas vítimas apresentavam-se com lesões neurológicas irreversíveis (doença de Minamata aguda e congênita). Contudo, o que ocorreu nos primeiros anos após a exposição, não ficou definitivamente esclarecido ${ }^{61014}$.

A ingestão diária aceitável de metilmercúrio em peixes ainda continua a ser discutida, pois níveis elevados de mercúrio total, de até $24 \mu \mathrm{g} / \mathrm{g}^{-1}$, foram encontrados em peixes consumidos pela população indígena do Canadá, as quais apresentaram elevadas concentrações de metilmercúrio no sangue $\left(20 \mathrm{a} 660 \mu \mathrm{g} / \mathrm{g}^{-1}\right)$, entretanto, não permitiram a caracterização definitiva de casos de intoxicação ${ }^{312} 15$.

Contudo, observou-se o aparecimento de manifestações clínicas da intoxicação por metilmercúrio, em pescadores e familiares do Rio Sunhai (China), que apresentavam concentrações médias de mercúrio total no cabelo de $19,2 \mu \mathrm{g} / \mathrm{g}^{-1}$ variando de $5,3 \mu \mathrm{g} / \mathrm{g}^{-1}$ a $71,2 \mu \mathrm{g} / \mathrm{g}^{-1}$ com predomínio de metilmercúrio ${ }^{13}$. Tais valores são comparáveis com os encontrados neste estudo nas populações ribeirinhas do Tapajós.

Os resultados obtidos neste estudo demonstraram que:

a) a contaminação mercurial nas comunidades ribeirinhas do rio Tapajós, confirmam os achados de outros estudos;

b) a manutenção da exposição humana ao metilmercúrio é, provavelmente, pela ingestão dos peixes contaminados;

c) a contaminação humana pelo mercúrio se mantém estável ao longo do período estudado;

d) as concentrações de mercúrio total e metilmercúrio observadas nas amostras de cabelo das populações ribeirinhas estudadas, admitem a possibilidade do aparecimento de sinais e sintomas da intoxicação.

Portanto, os dados obtidos recomendam a manutenção da monitorização do mercúrio total e do metilmercúrio em amostras de cabelo e estudos de natureza clínico-epidemiológico nas áreas avaliadas com vistas a implantação de medidas de controle da contaminação e prevenção dos danos causados pelo metilmercúrio, particularmente nos grupos de risco (gestantes e crianças).

\section{REFERÊNCIAS BIBLIOGRÁFICAS}

1. Akagi $\mathrm{H}$, Malm O, Branches FJP. Human exposure to mercury due to gold mining in the Amazon, Brazil. A review. Environmental Science 4:199-211, 1996.

2. Akagi $\mathrm{H}$, Malm O, Kinjo $\mathrm{Y}$, Harada M, Branches FJP, Pfeiffer WC, Kato $\mathrm{H}$. Methylmercury pollution in the Amazon, Brazil. The Science of Total Environment 175:85-95, 1995.

3. Clarkson TW. Recent advances in the toxicology of mercury with emphasis on the arylmercurials. Critical Reviews in Toxicology 1:203-234, 1972.
4. D'itri FM. The environmental mercury problem. CRCPress, Michigan, 1972.

5. Grandjean P, Cardoso B, Guimarães G. Mercury poisoning. The Lancet 342:991,1993.

6. Harada M. Minamata disease: methylmercury poisoning in Japan caused by environmental pollution. Critical Reviews in Toxicology 25:1-24, 1995.

7. Harada M. Neurotoxicity of methylmercury: Minamata and the Amazon. In: Yasui M, Strong MJ, Ota KK, Verity MA (eds) Mineral and metal neurotoxicology, CRC Press, New York, p.177-187,1997. 
8. Igata A. Epidemiological and clinical features of Minamata Disease. In: Suzuki T, Imura N, Clarkson TW (eds) Advances in mercury toxicology. Plenum Press, New York, p. 439-457, 1991.

9. Malm O, Pfeiffer WC, Souza CMM, Reuther R. Mercury pollution due to gold mining in the Madeira river basin, Brazil. Ambio 19:11-15, 1990.

10. Mariayama $H$, Tretatsuka M, Kinjo Y. Faetal Minamata Disease. A review. Environmental Sciences 3:15-23, 1994.

11. Pfeiffer WC, Lacerda LD. Mercury inputs into the Amazon region, Brazil. Environmental Technology Letters 9:325330, 1988.

12. Rose D. Mercury in man. In: Canada National Research Council (ed) Effects of mercury in the Canadian environment. Ottawa (NRCC no 16739) p.137-167, 1977.
13. Soong TR, Chu FR, Fun ZT. Epidemiological study on the health of residents along the Sunhua River, polluted by methylmercury. Environmental Sciences 3:41-54, 1994.

14. Takeuchi T, Eto K. Minamata disease, chronic occurrence from pathological viewpoints. In: Japan Environmental Agency (ed) Studies on the health effects of alkylmercury in Japan, Tokyo, p. 28-62, 1975.

15. Wheatley B. Exposure of Canadian aboriginal peoples to methylmercury. Environmental Science 3:3340,1994.

16. World Health Organization. Mercury. (Environmental Health Criteria, no 1). Geneva, 1976.

17. World Health Organization. Methylmercury. (Environmental Health Criteria, no 118). Geneva, 1990. 